\title{
Difficulty of Visual Search Modulates Neuronal Interactions and Response Variability in the Frontal Eye Field
}

Jeremiah Y. Cohen ${ }^{1}$, Pierre Pouget ${ }^{2,3,4}$, Geoffrey F. Woodman ${ }^{2,3,4}$, Chenchal R.

Subraveti ${ }^{2}$, Jeffrey D. Schall ${ }^{1,2,3,4}$, and Andrew F. Rossi ${ }^{1,2,3,4}$

\author{
${ }^{1}$ Vanderbilt Brain Institute, ${ }^{2}$ Department of Psychology, Vanderbilt University, ${ }^{3}$ Center for \\ Integrative and Cognitive Neuroscience, Vanderbilt University, ${ }^{4}$ Vanderbilt Vision \\ Research Center, Nashville, TN
}

Running head: Neuronal interactions in the FEF

Dr. Andrew F. Rossi

301 Wilson Hall

11121 st Avenue South

Vanderbilt University

Nashville, TN 37240

Phone: (615) 322-7466

FAX: (615) 343-8449

Email: andrew.rossi@vanderbilt.edu 


\begin{abstract}
The frontal eye field (FEF) is involved in selecting visual targets for eye movements. To understand how populations of FEF neurons interact during target selection, we recorded activity from multiple neurons simultaneously while macaques performed two versions of a visual search task. We used a multivariate analysis in a point process statistical framework to estimate the instantaneous firing rate and compare interactions among neurons between tasks. We found that FEF neurons were engaged in more interactions during easier visual search tasks as compared to harder search tasks. In particular, eye-movement-related neurons were involved in more interactions than visual-related neurons. In addition, our analysis revealed a decrease in the variability of spiking activity in the FEF beginning about 100 ms prior to saccade onset. The minimum in response variability occurred about $20 \mathrm{~ms}$ earlier for the easier search task compared to the harder one. This difference is positively correlated with the difference in saccade reaction times for the two tasks. These findings demonstrate that a multivariate analysis can provide a measure of neuronal interactions and characterize the spiking activity of FEF neurons in the context of a population of neurons.
\end{abstract}




\section{Introduction}

Neuronal activity in the primate frontal eye field (FEF) reflects visual target selection and eye movement commands (Thompson et al. 1996; Thompson et al. 1997; Bichot and Schall 1999; Schall and Thompson 1999; Murthy et al. 2001). Information from the visual system converges on the FEF (Schall et al. 1995b), and is integrated into eye movement commands via inputs to oculomotor structures (Hanes and Schall 1996, Sommer and Wurtz 1998; Sommer and Wurtz 2001; Helminski and Segraves 2003). Three functional classes of neurons have been described in the FEF: visual-related, visual-and-movement-related, and movement-related (Bruce and Goldberg 1985; Segraves and Goldberg 1987; Schall 1991; Schall and Hanes 1993; Schall et al. 1995a; Schall and Thompson 1999). How these classes of neurons in the FEF interact to contribute to target selection remains unknown. To address how such visual-to-motor integration occurs, we analyzed the activity of FEF neurons recorded during a visual search task, emphasizing interactions that occurred among simultaneously recorded neurons. We compared neuronal interactions associated with hard and easy visual search tasks. A hard task was defined as one with a high degree of similarity between the target of the search and the distractor stimuli to be ignored. An easy task was defined as one with a low degree of target-distractor similarity (Duncan and Humphreys 1989).

Recent developments in multivariate point process modeling of neural responses have provided an analytical framework to characterize neural activity in the context of interactions between simultaneously recorded neurons (Brown et al. 2002; Okatan et al. 2005; Truccolo et al. 2005). This analysis models the instantaneous firing rate of a neuron using its own spiking history and that of other simultaneously recorded neurons as covariates in the model. The significance of each covariate provides an estimate of its contribution to the response of the modeled neuron. If the multivariate model's 
estimate of instantaneous firing rate is significantly improved by including covariates representing the activity of other neurons, it is evidence of interactions among neurons in the recorded ensemble. Such interactions may be direct synaptic connections between neurons or may be mediated polysynaptically or by shared input. Compared to conventional univariate estimates of neuronal activity, such as the peristimulus time histogram (PSTH), the multivariate approach can distinguish between a neuron's response and its response in the context of interactions in a population of neurons. Furthermore, point process modeling of neural activity preserves spike timing information which is distorted by measures that average over time-interval windows (e.g., PSTH). Traditional approaches for analysis of interactions between neurons, such as the covariogram and joint peristimulus time histogram (Aertsen et al. 1989; Brody 1999b; Brody 1999a; Constantinidis et al. 2001), are limited to pairwise comparisons and do not provide adequate measures of ensemble interactions. The multivariate point process model estimates the instantaneous firing rate of a neuron in real time without limits on ensemble size. Nonetheless, it can extract the same interactions as pairwise measures.

We demonstrate that the multivariate approach can accurately model spiking activity in the FEF and characterize interactions among simultaneously recorded neurons while monkeys perform a visual search task. To better understand how neuronal interactions in the FEF contribute to target selection, we addressed the following questions: (1) Do interactions among FEF neurons differ for hard and easy search? (2) Are there differences in interactions between the three classes of FEF neurons? (3) Are interactions of movement-related neurons associated with saccade onset times?

\section{Methods}

\section{Behavioral task and recording}


Activity of FEF neurons was recorded in macaques performing a visual search task in which they were required to saccade to a singleton target defined by color (Sato et al. 2001). Each trial began with the monkey fixating a central spot for approximately 600 ms. A target was then presented at one of eight iso-eccentric locations equally spaced around the fixation spot (see inset, Figure 1A). The other seven locations contained distractor stimuli. Monkeys were given a juice reward for making a saccade to the target location and holding their gaze on the target for approximately $400 \mathrm{~ms}$. There were two levels of task difficulty, "hard" and "easy," determined by the degree of target-distractor similarity. The hard task contained a green target among yellow-green distractors. The easy task contained a green target among red distractors. Recordings were made simultaneously from 2-4 tungsten electrodes placed in the rostral bank of the arcuate sulcus. A neural ensemble was defined as a set of simultaneously recorded neurons with overlapping receptive fields. Our data set consisted of 91 neurons in 29 ensembles from one monkey (Macaca mulatta) and 21 neurons in 7 ensembles from a second monkey (Macaca radiata). Of the 36 ensembles, 15 contained 2 neurons, 10 contained 3 neurons, 6 contained 4 neurons, 4 contained 5 neurons, and 1 contained 8 neurons. Spikes were sorted offline using principal components analysis (Plexon, Inc.). Because data from each monkey were collected during the same behavioral tasks and were similar, we pooled data from both monkeys.

Monkeys were trained on a memory-guided saccade task to distinguish visual- from movement-related activity (Hikosaka and Wurtz 1983; Bruce and Goldberg 1985). The target was flashed alone for $80 \mathrm{~ms}$. The monkey was required to maintain fixation for 400-1000 ms after the target offset. When the fixation spot disappeared, the monkey was rewarded for a saccade to the remembered location of the target. The movementrelated neurons analyzed here had significantly greater responses in the 100 ms leading up to the saccade than in the $100 \mathrm{~ms}$ after the target flash. Visual- and visual-and- 
movement-related neurons had greater responses in the $100 \mathrm{~ms}$ after the target flash than in the $100 \mathrm{~ms}$ before the target flash.

Each monkey was surgically implanted with a head post, a scleral eye coil, and a recording chamber. Surgery was conducted under aseptic conditions with isofluorane anesthesia (see Schall et al. 1995a). Antibiotics and analgesics were administered postoperatively. All experimental procedures were performed in accordance with the National Institutes of Health Guide for the Care and Use of Laboratory Animals and approved by the Vanderbilt Institutional Animal Care and Use Committee.

\section{FIGURE 1 HERE}

\section{Data analysis}

To assess simultaneously the interactions of several neurons, we used a point process multivariate analysis (Okatan et al. 2005; Truccolo et al. 2005). The point process framework can provide for comparisons of arbitrarily large ensembles of simultaneously recorded neurons that pairwise measures cannot. We constructed a statistical model of the firing rate of a neuron by incorporating its firing history and the firing history of other neurons in its ensemble from stimulus onset to saccade onset (5315 correct hard search trials, 7414 correct easy search trials). We used a modified version of the generalized linear model (GLM) approach recently applied by Truccolo et al. (2005). The modification was necessary because the fitted GLMs for the hard and easy tasks may be different. Therefore, unless they are nested, comparing such models statistically is intractable. We modified the GLM in the following way so that the data for both the hard and the easy tasks (for a particular neuron) were combined in a single GLM.

Using the theory of point processes, we represented recorded spike trains as sets of discrete event times. We modeled the instantaneous firing rate (conditional intensity 
function) of a neuron as a combination of terms of covariates (Truccolo et al. 2005). The conditional intensity function $\left(\lambda_{t}\right)$ is more informative of the instantaneous firing rate than univariate measures (e.g., PSTH) because its estimate is derived in the context of interacting neurons. We predicted the firing of a neuron using its firing history (autoregressive process) and the firing history of other neurons recorded simultaneously (crossregressive process). We concatenated data from all correct trials for each neuron and used a generalized linear model (GLM) to predict the firing rate of a neuron as

$\log \left(\lambda_{t}\right)=\left(\mu x_{1}+\varphi x_{2}\right)+\sum_{i=1}^{Q}\left(\alpha_{i} x_{1}+\beta_{i} x_{2}\right) \delta N_{t-i}+\sum_{c} \Sigma_{j=1}{ }^{R}\left(\eta^{(c)}{ }_{j} x_{1}+v^{(c)}{ }_{j} x_{2}\right) \delta N^{(c)}{ }_{t-j}$,

where $\lambda_{t}$ is the firing rate at time $t, \mu$ is a baseline term associated with the hard search condition, $\varphi$ is a baseline term associated with the easy search condition, $\left\{\alpha_{i}\right\}$ is the set of hard search autoregressive (AR) parameters, $\left\{\beta_{i}\right\}$ is the set of easy search AR parameters, $\left\{\eta_{j}\right\}$ is the set of hard search crossregressive $(C R)$ parameters (one for each neuron in the ensemble at each lag), $\left\{V_{j}\right\}$ is the set of easy search CR parameters, and $\delta \mathrm{N}^{(\mathrm{c})}{ }_{\mathrm{t}-\mathrm{k}}$ is the spike count in the $k^{\text {th }}$ ms prior to the current time $t$, for neuron $c$ in the ensemble. $Q$ and $R$ are the autoregressive and crossregressive lags of the model, respectively. The indicator variables $\mathrm{x}_{1}$ and $\mathrm{x}_{2}$ combine the parameters associated with each task into a single model. $x_{1}$ is 0 for easy search trials and 1 for hard search trials. $x_{2}$ is 1 for easy search trials and 0 for hard search trials. Because of constraints on the length of the recordings (relative to the firing rate) we set $Q=R=30$. This constraint was not of consequence to our analysis because the parameter fits stabilized well before lags of $30 \mathrm{~ms}$. In the GLM above, the AR parameters describe the timing of the modeled neuron's dependence on its firing history and the CR ones describe the timing of interactions between neurons. To compare models of hard and easy visual search trials, $\mathrm{x}_{1}$ and $\mathrm{x}_{2}$ terms were merged to create a separate GLM for each task. 
We fit the GLM using an iteratively reweighed least squares algorithm (McCullagh and Nelder 1989). This algorithm provides a robust maximum likelihood estimate of model parameters. If the assumptions of the GLM are met, then the fitted model's residuals should have a normal distribution around mean 0 and constant variance with no autocorrelations (McCullagh and Nelder 1989; Truccolo et al. 2005). Thus, we examined the residuals of each of our model fits.

To compare the fit of nested models, we used likelihood ratio tests. For each neuron, we compared the model deviance $(D=-2 \log L)$ for AR-only models and for AR-CR models for hard and easy search trials separately. This deviance comes from a $x^{2}$ distribution. Thus, we can test the hypothesis that adding $\mathrm{CR}$ terms to an AR model does not improve the GLM fits (McCullagh and Nelder 1989). If the likelihood ratio is large, the modeled neuron's response depends heavily on the ensemble neurons' responses. We measured variability in $\lambda_{t}$ across neurons using a standardized measure of variability, the coefficient of variation (sd/mean). All analyses were performed in $R(R$ Development Core Team 2006).

\section{Model fits}

To demonstrate that the GLM accurately accounted for the firing of FEF neurons, we compared the conditional intensity functions $\left(\lambda_{t}\right.$, instantaneous firing rates) against the observed spike trains. Figure 2A shows the modeled intensity (black curve) and the observed spike train (gray vertical lines) for one example neuron over the course of 300 ms. The model covariates are the neuron's spiking history and the history of a second neuron in the ensemble. The modeled rate closely follows the observed spike times. Note that the magnitude of the intensity increases with the frequency of spikes. The brief (1 ms) decrease in the intensity following a spike likely corresponds to the neuron's absolute refractory period (Truccolo et al. 2005). This decrease is evident in Figure 2B, 
which depicts the spike-triggered average intensity for the neuron in Figure 2A. We also examined the Pearson residuals for each model. If the Pearson residuals were distributed normally with mean 0 and variance 1 , then the firing left unexplained by the model was insignificant (McCullagh and Nelder 1989). For the model in Figure 2, which was representative of the model fits of FEF neurons in our sample, the mean of the Pearson residuals was $6.08 \cdot 10^{-4}$, the variance was 0.892 , and they were distributed normally. Models for all neurons had normally distributed Pearson residuals (ShapiroWilk test, $p<0.05)$.

\section{FIGURE 2 HERE}

\section{Simulation and interpretation of parameters}

A simulation was performed to test whether the GLM analysis extracted the same interactions that standard measures of pairwise interactions do. Figures $3 \mathrm{~A}$ and $\mathrm{B}$ illustrate a comparison of the GLM fitting results for a simulated pair of spike trains with a standard measure of interactions between pairs of neurons, the covariogram (Brody 1999a). The CR parameter values in Figure 3B match the lags of high correlation between the pair of simulated spike trains in Figure 3A. Significant parameters (by Wald $Z$ test, $p=0.05$ ) corresponded to interactions between the covariate and the target neuron at that lag, though not necessarily "monosynaptically." Positive parameter values corresponded to high probability that the covariate neuron excited the modeled neuron. Negative values corresponded to high probability of inhibition. Nonsignificant parameters (gray points) corresponded to low probability that the covariate interacted with the modeled neuron at those lags.

Figures $3 C$ and $D$ show boxplots of parameter values at each time lag for all hard and easy models for all FEF recordings. Both AR and CR parameter values were stable past 
$15 \mathrm{~ms}$, making it unnecessary to use a history of greater than $30 \mathrm{~ms}$. The neurons' own history-dependence (AR parameters) typically included about $10 \mathrm{~ms}$ and was inhibitory (negative parameter values). This likely corresponded to effects of absolute and relative refractory periods (Truccolo et al. 2005). Neurons' dependence on ensemble neurons' history (CR parameters) was, overall, relatively uniform except for at a lag of $1 \mathrm{~ms}$. Baseline rates differed by less than one spike per second between hard and easy tasks.

\section{FIGURE 3 HERE}

There were no significant differences between AR or CR parameters between hard and easy tasks. Figures $3 C$ and $D$ display the overall pattern of history dependence on a neuron's firing.

\section{Results}

Activity of 112 neurons was recorded in the FEF of two macaques performing two versions of a saccade-to-oddball visual search task (see inset, Figure 1A). These neurons were recorded in 36 ensembles. An ensemble was defined as a set of simultaneously recorded neurons with overlapping receptive fields. Ensemble sizes ranged from two to eight neurons.

Because both monkeys showed similar differences in performance for the easy and hard search tasks, these data were pooled. The easy and hard search tasks resulted in significant differences in percent correct (hard task $=71.6 \%$; easy task $=96.6 \%$;

Wilcoxon rank sum test, $p<10^{-15}$ ) and mean saccade reaction time (hard task $=237 \mathrm{~ms}$; easy task $\left.=196 \mathrm{~ms} ; p<10^{-15}\right)$. Figure $1 \mathrm{~A}$ shows the densities of saccade reaction times (RT) for the hard and easy search tasks. In addition to the significant difference in the mean RT (44.3 ms) for the two tasks, the variability of saccade RT was greater for the 
hard search task $(s d=65.3 \mathrm{~ms})$ than the easy search task $(\mathrm{sd}=41.2 \mathrm{~ms})$. The mean difference in reaction time (hard minus easy) within each session was $44.4 \pm 3.6 \mathrm{~ms}$ (mean $\pm \mathrm{se}$ ), with a minimum of $15.4 \mathrm{~ms}$ (Figure 1B).

\section{Do interactions among FEF neurons differ for hard and easy search?}

We asked whether the amount of interactions among neurons in the FEF was affected by the difficulty of the visual search task. To measure interactions between neurons, we used a point process multivariate analysis to model the instantaneous firing rate of each neuron for each task (hard and easy) taking into account the firing history of all neurons in the recorded ensemble (see Methods). The model estimated the conditional intensity function (instantaneous firing rate) of the neuron with respect to the covariates (autoregressive, AR, and crossregressive, CR) when a saccade was made to a target located within the receptive field. We computed the likelihood ratios of models for each neuron for each task by subtracting the deviance of the AR-CR model from the deviance of the AR model. Large likelihood ratios indicated that including ensemble neurons in the model of a neuron greatly improved the prediction of the firing of that neuron.

Because the likelihood ratio is distributed as $x^{2}$, it is an ideal measure of the degree to which addition of CR covariates improved the estimate of the firing rate (McCullagh and Nelder 1989).

We found that for $63.7 \%$ of easy task models and $51.6 \%$ of hard task models, $x^{2} p$ values were less than 0.05 , indicating that the inclusion of CR covariates improved the prediction of firing rate in the majority of models. We then compared the likelihood ratios of the two pairs of models (hard versus easy) to determine under which task the addition of ensemble responses (CR covariates) improved the model the most. The model that benefited the most from addition of CR covariates was judged to convey more interactions. Figure $4 \mathrm{~A}$ is a histogram of the difference between likelihood ratios for 
each neuron (easy minus hard). The histogram is shifted significantly to the right of zero (paired Wilcoxon rank sum test, $p=3.05 \cdot 10^{-3}$ ). Thus, we could better predict the firing of neurons in the easy task than the hard task when including the firing history of other neurons recorded simultaneously, indicating that neurons interacted more with each other during easy visual search. Simulations revealed that, regardless of visual search task, adding randomly firing simulated neurons to models of FEF neurons did not improve those model fits. Therefore, improvements to models of FEF neurons by adding CR covariates were due to neuronal interactions, whether monosynaptic, polysynaptic, or via shared input. There was no significant correlation $(p=0.490)$ between RT difference (hard minus easy) and likelihood ratio difference (easy minus hard), possibly due to low variability in RTs.

\section{FIGURE 4 HERE}

We cannot completely rule out that differences in interactions were not due to differences in trial lengths or spike counts between hard and easy trials. This seems unlikely, however, for two reasons: First, the average firing rate difference between hard and easy trials was less than one spike per second. Second, an analysis in which we equated for trial length yielded similar results. In this analysis, we removed data from the end of the spike train for each trial to equate with the length of the spike train of the shortest trial in each session. Before equating for trial length, $63.7 \%$ of neurons in the easy task and $51.6 \%$ in the hard task showed improved fits upon addition of CR covariates, a difference of $12.1 \%$. After equating for trial length, the number of neurons that showed improved fits decreased due to loss of data, but the same trend remained: $31.9 \%$ of neurons in the easy task and $20.9 \%$ in the hard task showed improved fits upon addition of CR covariates, a difference of $11.0 \%$. Thus, despite discarding a 
considerable amount of data to equate for trial length, we found that neurons exhibited more interactions during easy trials than during hard trials.

\section{Are there differences in interactions between the three classes of FEF neurons?}

We classified each neuron as visual-related, movement-related, or visual-andmovement-related. There were 46 visual-related neurons, 16 movement-related neurons, 49 visual-and-movement-related neurons, and one that was unclassified. Figure 5 shows the responses of representative neurons of each class from our sample of recordings. The model for each showed improved fit upon addition of CR covariates. Each peristimulus time histogram (PSTH) in this figure illustrates the average firing rate of the neuron when the target was presented in the neuron's receptive field. Visualrelated neurons had clear responses about 50 ms after target onset (Figure 5A), visualand-movement-related neurons had similar visual latencies and increased firing leading up to saccades (Figure 5B), and movement-related neurons fired at baseline until about $60 \mathrm{~ms}$ before saccades (Figure 5C). The difference in visual-and-movement- and movement-related responses during the hard and easy tasks correlated with the difference in mean RT between the hard (gray arrowhead) and easy (black arrowhead) tasks.

\section{FIGURE 5 HERE}

We tested whether there were systematic differences in interactions based on neuron class. Figure 4B shows likelihood ratio differences (easy minus hard) by neuron class. There were no significant differences in the distributions of likelihood ratio differences between visual- and movement-related neurons (Wilcoxon rank sum test, $p=0.203$ ), visual- and visual-and-movement-related neurons $(p=1.00)$, and visual-and-movement- 
and movement-related neurons $(p=0.329)$. Figures $4 C$ and $D$ show likelihood ratios for easy and hard tasks split by neuron class. We found that the likelihood ratios were significantly larger for movement-related neurons (white bars) than for visual-related neurons (black bars) for both hard (Figure 4D; $p=1.90 \cdot 10^{-3}$ ) and easy tasks (Figure $\left.4 \mathrm{C} ; p=2.18 \cdot 10^{-5}\right)$. Likewise, the ratios were larger for visual-and-movement-related neurons (gray bars) than for visual-related neurons for both tasks (hard: $p=6.34 \cdot 10^{-12}$; easy: $p=7.36 \cdot 10^{-11}$ ). This indicates that movement- and visual-and-movement-related neurons were engaged in greater interactions than the visual-related neurons.

Likelihood ratios were significantly larger for the easy task than the hard task for visualrelated neurons (paired Wilcoxon rank sum test, $p=0.0425$ ) and movement-related neurons $(p=0.0443)$ and approached significance for visual-and-movement-related neurons $(p=0.0990)$. This indicates that the model better predicted the firing of neurons in the easy task upon addition of CR covariates than the hard task and that addition of CR covariates significantly improved firing predictions. The percent of neurons of each class with improved fits upon addition of CR covariates was larger for neurons with movement-related activity than for neurons with only visual-related activity. For the easy task, $48.0 \%$ of visual-related neurons, $87.5 \%$ of movement-related neurons, and $63.3 \%$ of visual-and-movement-related neurons showed improved fits. For the hard task, $32.0 \%$ of visual-related neurons, $56.3 \%$ of movement-related neurons, and $59.2 \%$ of visual-and-movement-related neurons showed improved fits. There were no differences in mean firing rate between neurons that showed improved fits and those that did not for each neuron class (Wilcoxon rank sum test, $p>0.287$ ).

\section{Are interactions among FEF neurons time-locked to saccade onset?}

Because movement-related neurons in the FEF characteristically increase their firing leading up to saccades (Hanes and Schall 1996; see example Figure 5C), we analyzed 
the conditional intensity functions $\left(\lambda_{t}\right.$, instantaneous firing rates) for movement-related neurons to determine if the timing of interactions reflected the decision of the monkeys to move their eyes. To observe the neuronal responses around saccades, we modeled the intensity of these neurons from target onset to $50 \mathrm{~ms}$ after the saccade. Figure 6 shows the mean intensity $\left(\lambda_{t}\right)$ for the 16 movement-related neurons during the hard task (Figure $6 \mathrm{~A}$ ) and during the easy task (Figure 6B). The gray curves show the mean intensities of AR models during the 100 ms leading up to saccades for the hard task, the black curves the mean intensities of AR-CR models.

\section{FIGURE 6 HERE}

Figures $6 C$ and $D$ show the difference between AR-CR and AR models of mean intensities for hard trials (C) and easy trials (D). For both hard and easy tasks, addition of CR covariates increased $\lambda_{t}$ leading up to saccades, relative to $\lambda_{t}$ for AR models. Thus, interactions between neurons statistically accounted for a significant portion of the presaccadic activity in movement-related neurons. Figures $6 \mathrm{E}$ and $\mathrm{F}$ show the normalized mean PSTH (gray curve) and intensity function (black curve) for hard trials (E) and easy trials (F). A comparison of the intensity functions and PSTHs shows that the AR-CR models accurately describe both the magnitude and the dynamics of the PSTHs for the two search tasks. The intensity functions are shifted about $30 \mathrm{~ms}$ to the right of the PSTHs, reflecting the integration of $30 \mathrm{~ms}$ of firing history into the estimate of the intensities.

To further explore the effect of ensemble interactions on the activity of movementrelated neurons, we compared the variability of the conditional intensity functions for each search task. The coefficient of variation $(\mathrm{CV}, \mathrm{sd} / \mathrm{mean})$ is a measure of standardized variability frequently used in neuroscience and may be interpreted as a 
“noise-to-signal” ratio (Stein and Matthews 1965; de Ruyter van Steveninck et al. 1997; Stevens and Zador 1998; Feng and Brown 1999). We used the CV to measure changes in instantaneous firing rate of movement-related neurons that occurred just before saccades. Decreases in the CV over time reflected less variability (or noise) in the system.

Figure 7 shows the $\mathrm{CV}$ of the mean intensity function for the movement-related neurons for the hard (gray curves) and easy (black curves) tasks for AR-CR models (Figure 7A) and AR models (Figure 7B). For both hard and easy tasks, the CV decreased leading up to saccades in the AR-CR models until just before saccades, at which point the CV increased. Thus, addition of CR covariates decreased standardized variability (CV) leading up to saccades.

\section{FIGURE 7 HERE}

Remarkably, there was a clear difference between the time at which the CV began to increase preceding saccades for hard and easy tasks. We fit smoothing splines to each curve to estimate the time at which each curve attained its minimum. In the AR-CR models, the CV started increasing $28 \mathrm{~ms}$ before saccades for the easy task and $7 \mathrm{~ms}$ for the hard task. This difference suggests that the movement-related neurons responded with the least variability about $20 \mathrm{~ms}$ earlier in the easy task, despite the fact that we aligned intensities on saccade times. The difference in minimum CV times between easy and hard search was closer for AR-CR models (Figure 7A) than for AR models (Figure 7B). Presumably, this was due to poorer fits of AR models. It is important to note that, although intensities were shifted to the right of PSTHs, the timing results in Figure 7 compare identical measures to each other (i.e., intensity to intensity and PSTH 
to PSTH). Thus, the differences in time course of the CV between the hard and easy tasks reflect the dynamics of the conditional intensity functions.

We compared the results using the intensity function to a standard model of the firing rate, the PSTH. Figure $7 \mathrm{C}$ shows the $\mathrm{CV}$ of the PSTHs of movement-related neurons. Similar to the results obtained from the intensity functions (Figures 7A and B), the PSTH CV decreased leading up to saccades and increased 5-10 ms before saccades. However, there was no difference between time of increase for hard and easy tasks. It is important to keep in mind that comparing changes in the PSTH between hard and easy tasks cannot reveal the same information as the intensity can, because the latter measures changes in the system in the context of interacting neurons. Thus, the intensity function is a better measure of instantaneous firing rate than the PSTH to measure time of response variability in the FEF.

\section{Discussion}

An outstanding problem in neuroscience is determining how populations of neurons interact to produce behavior. We have recorded simultaneous activity from multiple frontal eye field (FEF) neurons while monkeys performed two versions of a visual search task, one hard and one easy, defined by similarity between the target and distractors.

The present results indicate that easier visual search tasks are associated with greater interactions among populations of FEF neurons. This is seemingly counterintuitive. After all, why should neurons interact more during a task that seemingly requires fewer resources to solve? The average firing rates are not significantly different between the two conditions, so there must be a difference in the structure of the spike trains. There are at least two potential explanations. (1) During hard tasks, FEF neurons fire less randomly, requiring fewer interactions to accomplish the same goals (target selection and saccade preparation). (2) FEF neurons fire more randomly during hard tasks, 
requiring more information from other neurons. We measured the coefficient of variation (CV) of the interspike intervals during hard and easy tasks. We found no significant difference between the two $(p=0.255)$, although there was a trend toward larger $\mathrm{CV}$ during the easy task. Further studies are required to determine whether FEF neurons' firing patterns differ between tasks.

Hanes and Schall (1996) determined that single movement-related neurons in the FEF could reliably predict saccade reaction time. This finding leads to the question of how movement-related neurons integrate visual information. It is possible that interactions between the three neuron classes in the FEF fully predict the timing of saccades. Simultaneous recordings from the FEF and other prefrontal areas (e.g., area 46) or parietal areas (e.g., lateral intraparietal cortex) would address this issue. In concert with a point process analysis, such an experiment would describe the timing of interactions between areas and determine how neurons in other cortical areas interact with FEF neurons to decide to move the eyes.

We propose that the timing of changes in the intensity function of movement-related neurons reveals the time at which networks of neurons decide to initiate a saccade. For models that include ensemble activity, the CV decreases until just before saccades, which coincides with the period of time when information about the decision to saccade accumulates. The CV is a second-order measure. As such, it reflects changes in noise in the system. Thus, it provides an estimate of the time between the saccade decision time of FEF movement-related neurons and execution of the saccade. Because the CV reflects noise in the system, remarkably, this also suggests that the noise in the system actually decreases as the firing rate of these neurons increases leading up to saccades (until the increase in $\mathrm{CV}$ just prior to saccades). Therefore, we show not only that movement-related neurons have activity sufficient to trigger a saccade (Hanes and 
Schall 1996), but show that their presaccadic activity reflects a decrease in firing variability.

The difference in time of increase of $\mathrm{CV}$ between hard and easy tasks has important implications for when the decision to make a saccade occurs. In our experiments, the neurons spent 20 ms longer reaching their minimum in response variability in the hard task than in the easy one. This may correspond to earlier decision times in the easy task than in the hard one. Therefore, it appears that the decision to move the eyes is mediated by the movement-related neurons. The larger likelihood ratios for movementrelated neurons versus visual-related neurons suggest that the movement-related neurons receive greater interactions from other FEF neurons. This agrees with models of visual search in the FEF, which assert that projections from visual-related neurons to movement-related neurons transform information about visual stimuli into a saccade execution plan (Thompson et al. 1996).

How does the estimate of 20 ms difference in time of minimum response variability correspond to differences in saccade reaction time (RT)? The mean RT difference between the hard and easy tasks was $44.3 \mathrm{~ms}$. The CV during the $100 \mathrm{~ms}$ after target onset did not decrease for visual-related neurons (data not shown). This may be due to the location of presynaptic neurons connecting with these visual-related neurons. If, as the anatomy suggests (Schall et al. 1995b), visual-related neurons receive synaptic connections from visually-responsive neurons in the parietal, temporal, and occipital cortices, then we would not have observed visual-related FEF neurons in the context of those interactions. Simultaneous recording from the FEF and cortical areas that project to the FEF would address this issue. Such an experiment would address whether sensorimotor integration occurs in a single bottom-up volley or is the result of continued flow of information between neurons with sensory responses and those with motor responses (Riehle et al. 1997; Woodman et al. 2007). 
We have shown that accounting for ensemble activity is a powerful method of modeling the firing rate of a neuron. There are several ways to measure activity of single neurons, however. A continuous function best represents the firing of a neuron if it accounts for the synaptic input to the neuron (a network property) and approximates the instantaneous firing rate of the neuron. The intensity function has two advantages over other measures of neuronal firing, such as the peristimulus time histogram (PSTH). First, it accounts for network activity (the crossregressive covariates) and second, it approximates instantaneous firing rate better than the PSTH (Truccolo et al. 2005). We have shown that using the intensity function to describe the firing of FEF neurons reveals the time course of activity leading to the decision to saccade more sensitively than the PSTH.

While the point process model can account for a large number of influences on a neuron's firing, it is not a mechanistic model. It cannot, therefore, distinguish between neurons that are synaptically connected and neurons that share common input. This limitation is shared by other common techniques such as the covariogram and joint peristimulus time histogram. A challenge for future studies of neuronal interactions is to include knowledge about anatomical connections and biophysical properties of neurons in mechanistic models of networks of neurons.

We postulate that the distinction between easy and hard tasks, as detected by our results (e.g., difference in lag between minimum CV and saccade) is continuous, rather than dichotomous. Theoretically, there must exist a minimal set of neurons that are required to complete a given visual search task and a latest time prior to saccade that the decision is made. Our results show that easier visual search tasks are associated with greater interactions among populations of FEF neurons and may result in earlier saccade decision times. 


\section{Acknowledgments}

We thank Dr. Yosef Cohen for helpful discussions. This work was supported by T32MH064913, R01-EY08890, P30-EY08126, P30-HD015052, the McKnight Endowment Fund for Neuroscience, and Robin and Richard Patton through the E. Bronson Ingram Chair in Neuroscience. 


\section{References}

Aertsen AM, Gerstein GL, Habib MK, Palm G. Dynamics of neuronal firing correlation: modulation of "effective connectivity". J Neurophysiol 61: 900-917, 1989.

Bichot NP, Schall JD. Effects of similarity and history on neural mechanisms of visual selection. Nat Neurosci 2: 549-554, 1999.

Brody CD. Correlations without synchrony. Neural Comput 11: 1537-1551, 1999a.

Brody CD. Disambiguating different covariation types. Neural Comput 11: 1527-1535, 1999b.

Brown EN, Barbieri R, Ventura V, Kass RE, Frank LM. The time-rescaling theorem and its application to neural spike train data analysis. Neural Comput 14: 325-346, 2002.

Bruce CJ, Goldberg ME. Primate frontal eye fields. I. Single neurons discharging before saccades. J Neurophysiol 53: 603-635, 1985.

Constantinidis C, Franowicz MN, Goldman-Rakic PS. Coding specificity in cortical microcircuits: a multiple-electrode analysis of primate prefrontal cortex. J Neurosci 21: 3646-3655, 2001.

\section{de Ruyter van Steveninck RR, Lewen GD, Strong SP, Koberle R, Bialek W.}

Reproducibility and variability in neural spike trains. Science 275: 1805-1808, 1997. 
Duncan J, Humphreys GW. Visual search and stimulus similarity. Psychol Rev 96: 433-458, 1989.

Feng JF, Brown D. Coefficient of variation of interspike intervals greater than 0.5. How and when? Biol Cybern 80: 291-297, 1999.

Hanes DP, Schall JD. Neural control of voluntary movement initiation. Science 274: 427-430, 1996.

Helminski JO, Segraves MA. Macaque frontal eye field input to saccade-related neurons in the superior colliculus. J Neurophysiol 90: 1046-1062, 2003.

Hikosaka O, Wurtz RH. Visual and oculomotor functions of monkey substantia nigra pars reticulata. III. Memory-contingent visual and saccade responses. J Neurophysiol 49: 1268-1284, 1983.

McCullagh P, Nelder JA. Generalized Linear Models. Boca Raton, FL: Chapman \& Hall/CRC, 2nd edition, 1989.

Murthy A, Thompson KG, Schall JD. Dynamic dissociation of visual selection from saccade programming in frontal eye field. J Neurophysiol 86: 2634-2637, 2001.

Okatan M, Wilson MA, Brown EN. Analyzing functional connectivity using a network likelihood model of ensemble neural spiking activity. Neural Comput 17: 1927-1961, 2005. 
R Development Core Team. R: A Language and Environment for Statistical Computing. R Foundation for Statistical Computing, Vienna, Austria. ISBN 3-90005107-0, 2006.

Riehle A, Kornblum S, Requin J. Neuronal correlates of sensorimotor association in stimulus-response compatibility. J Exp Psychol Hum Percept Perform 23: 1708-1726, 1997.

Sato T, Murthy A, Thompson KG, Schall JD. Search efficiency but not response interference affects visual selection in frontal eye field. Neuron 30: 583-591, 2001.

Schall JD. Neuronal activity related to visually guided saccades in the frontal eye fields of rhesus monkeys: comparison with supplementary eye fields. J Neurophysiol 66: 559_ 579, 1991.

Schall JD, Hanes DP. Neural basis of saccade target selection in frontal eye field during visual search. Nature 366: 467-469, 1993.

Schall JD, Hanes DP, Thompson KG, King DJ. Saccade target selection in frontal eye field of macaque. I. Visual and premovement activation. J Neurosci 15: 6905-6918, 1995a.

Schall JD, Morel A, King DJ, Bullier J. Topography of visual cortex connections with frontal eye field in macaque: convergence and segregation of processing streams. $J$ Neurosci 15: 4464-4487, 1995b. 
Schall JD, Thompson KG. Neural selection and control of visually guided eye movements. Annu Rev Neurosci 22: 241-259, 1999.

Segraves MA, Goldberg ME. Functional properties of corticotectal neurons in the monkey's frontal eye field. J Neurophysiol 58: 1387-1419, 1987.

Sommer MA, Wurtz RH. Frontal eye field neurons orthodromically activated from the superior colliculus. J Neurophysiol 80: 3331-3335, 1998.

Sommer MA, Wurtz, RH. Frontal eye field sends delay activity related to movement, memory, and vision to the superior colliculus. J Neurophysiol 85: 1673-1685, 2001.

Stein RB, Matthews PBC. Differences in variability of discharge frequency between primary and secondary muscle spindle afferent endings of the cat. Nature 208: 12171218, 1965.

Stevens CF, Zador AM. Input synchrony and the irregular firing of cortical neurons. Nat Neurosci 1: 210-217, 1998.

Thompson KG, Bichot NP, Schall JD. Dissociation of visual discrimination from saccade programming in macaque frontal eye field. J Neurophysiol 77: 1046-1050, 1997.

Thompson KG, Hanes DP, Bichot NP, Schall JD. Perceptual and motor processing stages identified in the activity of macaque frontal eye field neurons during visual search. J Neurophysiol 76: 4040-4055, 1996. 
Truccolo W, Eden UT, Fellows MR, Donoghue JP, Brown EN. A point process framework for relating neural spiking activity to spiking history, neural ensemble, and extrinsic covariate effects. J Neurophysiol 93: 1074-1089, 2005.

Woodman GF, Kang MS, Thompson KG, Schall JD. Visual search efficiency modulates the beginning of response preparation: Neurophysiological evidence for discrete flow. Psych Sci In Press, 2007. 


\section{Figure legends}

Figure 1:

Behavioral task and performance. A, The upper histogram shows the density of saccade reaction times (RTs) for the hard task (gray dashed line denotes mean). The lower histogram shows the density of RTs for the easy task (black dashed line denotes mean). The hard search task consisted of a green target among yellow-green distractors. The easy task consisted of a green target among red distractors. Data are pooled across all sessions for both monkeys. B, Histogram of session-by-session RT differences (hard minus easy).

\section{Figure 2:}

Example model fit. A, Spike train and modeled intensity for one FEF neuron over 300 ms. Gray vertical lines denote spike times. The black curve is the modeled conditional intensity. B, Spike-triggered average intensity for the neuron in A over the course of the entire recording.

\section{Figure 3:}

Model interpretation. A, Covariogram between a pair of simulated neurons. Dashed lines indicate significance. B, CR parameter values with standard errors (from Wald Z test) from the GLM fit for the same pair. The gray points are not significantly different from 0 . C, AR parameter values significantly different from 0 for hard and easy search models. The filled point is the mean, the horizontal bar is the median, the box delimits the interquartile range, and the whiskers extend to the point no more than 1.5 times the interquartile range. Outliers are not shown in the figure but are included in the analyses. D, The same for CR parameters. 
Figure 4:

Likelihood ratio analysis. A, Histogram of likelihood ratio (LR) differences (easy minus hard) for each neuron). B, Histogram of LR differences split by neuron class (black: visual-related neurons; gray: visual-and-movement-related neurons; white: movementrelated neurons). C, Histogram of LRs in easy task models split by neuron class. D, Histogram of LRs in hard task models split by neuron class.

\section{Figure 5:}

Example neurons of each class. A, PSTH for a visual-related neuron for the hard (gray) and easy (black) tasks aligned to array onset. B, The same for a visual-and-movementrelated neuron. $\mathrm{C}$, The same for a movement-related neuron. Bin size is $3 \mathrm{~ms}$. The black arrowhead denotes the mean saccade reaction time during the recording session for the easy task, the gray arrowhead for the hard task.

\section{Figure 6:}

Mean instantaneous firing rate of movement-related neurons prior to saccade. A, The black curve is the mean intensity function for the 16 movement-related neurons during the hard search task, averaged over all trials for AR-CR models. The gray curve is the same for AR models. The curves are aligned to each trial's saccade. B, The same for AR-CR models (black) and AR models (gray) for the easy task. C, The difference between AR-CR and AR intensities for the hard task, i.e., the difference between the black and gray curves in A. D, The difference between AR-CR and AR intensities for the easy task. E, Comparison of intensities and PSTHs. Normalized mean intensity function (black curve) and PSTH (gray curve, 1 ms bins) for the hard task. F, The same for the easy task. 
Figure 7:

Coefficient of variation of intensities and PSTHs. A, The gray curve is the coefficient of variation of the mean intensity functions for the movement-related neurons during the hard task, the black curve during the easy task, averaged over all trials for AR-CR models. The dashed curves are smoothing splines, used to estimate the minima of the curves. Arrowheads denote these minima. B, The same for AR models. C, The gray curve is the coefficient of variation of the mean PSTH during the hard task, the black curve during the easy task. 

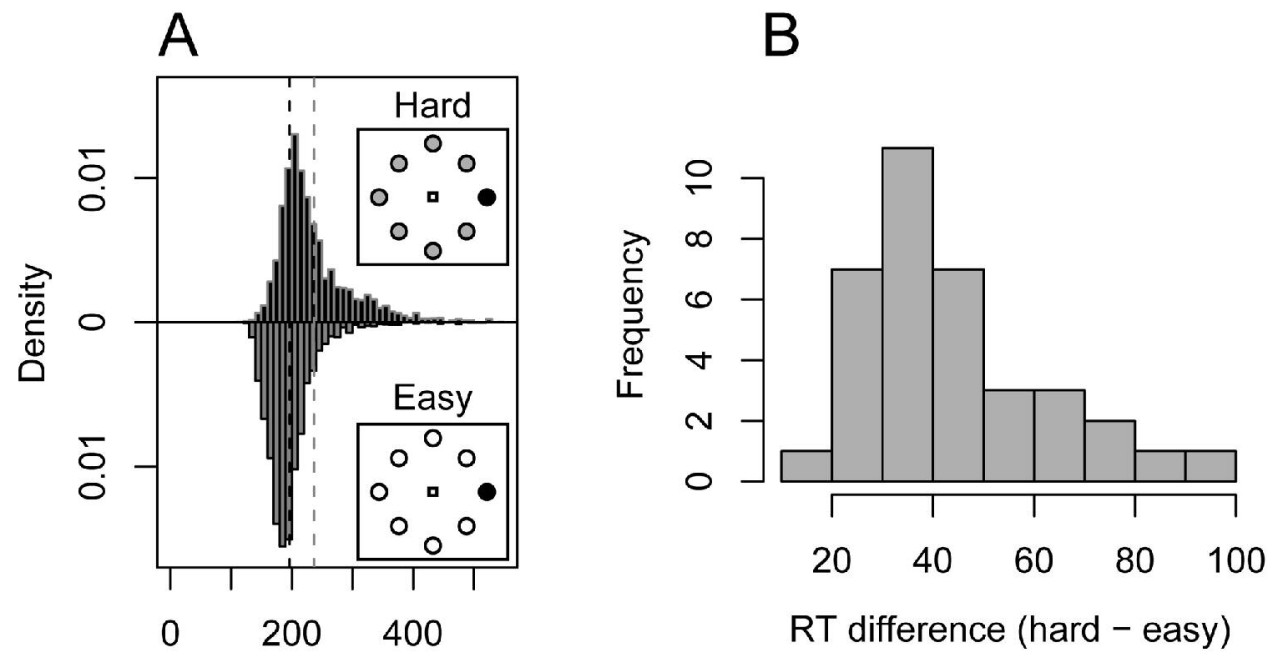

Saccade reaction time (ms)

Figure 1. Behavioral task and performance. A, The upper histogram shows the density of saccade reaction times (RTs) for the hard task (gray dashed line denotes mean). The lower histogram shows the density of RTs for the easy task (black dashed line denotes mean). The hard search task consisted of a green target among yellow-green distractors. The easy task consisted of a green target among red distractors. Data are pooled across all sessions for both monkeys. B, Histogram of session-by-session RT differences (hard minus easy).

$88 \times 51 \mathrm{~mm}(600 \times 600 \mathrm{DPI})$ 

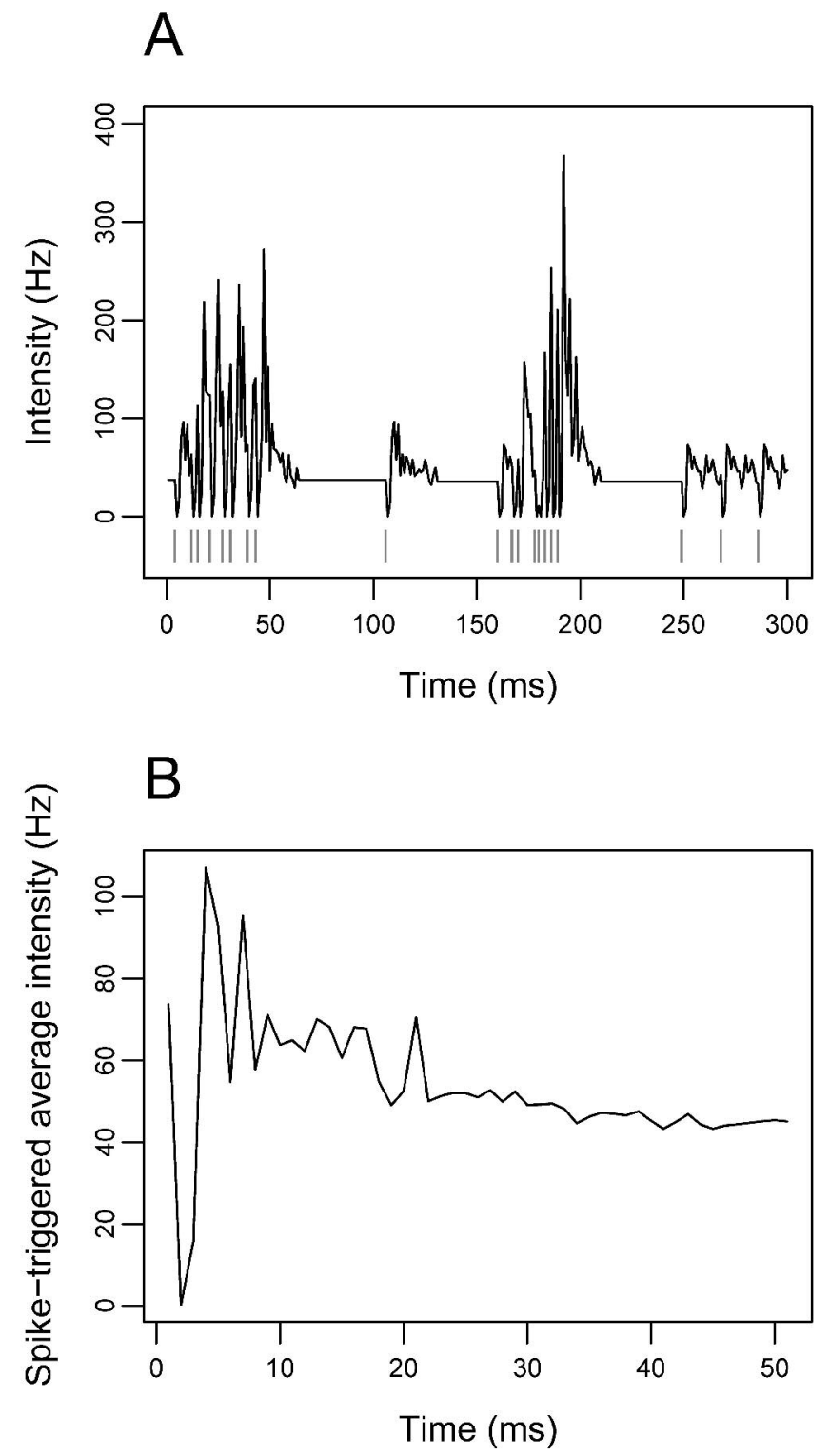

Figure 2. Example model fit. A, Spike train and modeled intensity for one FEF neuron over $300 \mathrm{~ms}$. Gray vertical lines denote spike times. The black curve is the modeled conditional intensity. B, Spike-triggered average intensity for the neuron in A over the course of the entire recording.

$177 \times 311 \mathrm{~mm}(600 \times 600 \mathrm{DPI})$ 

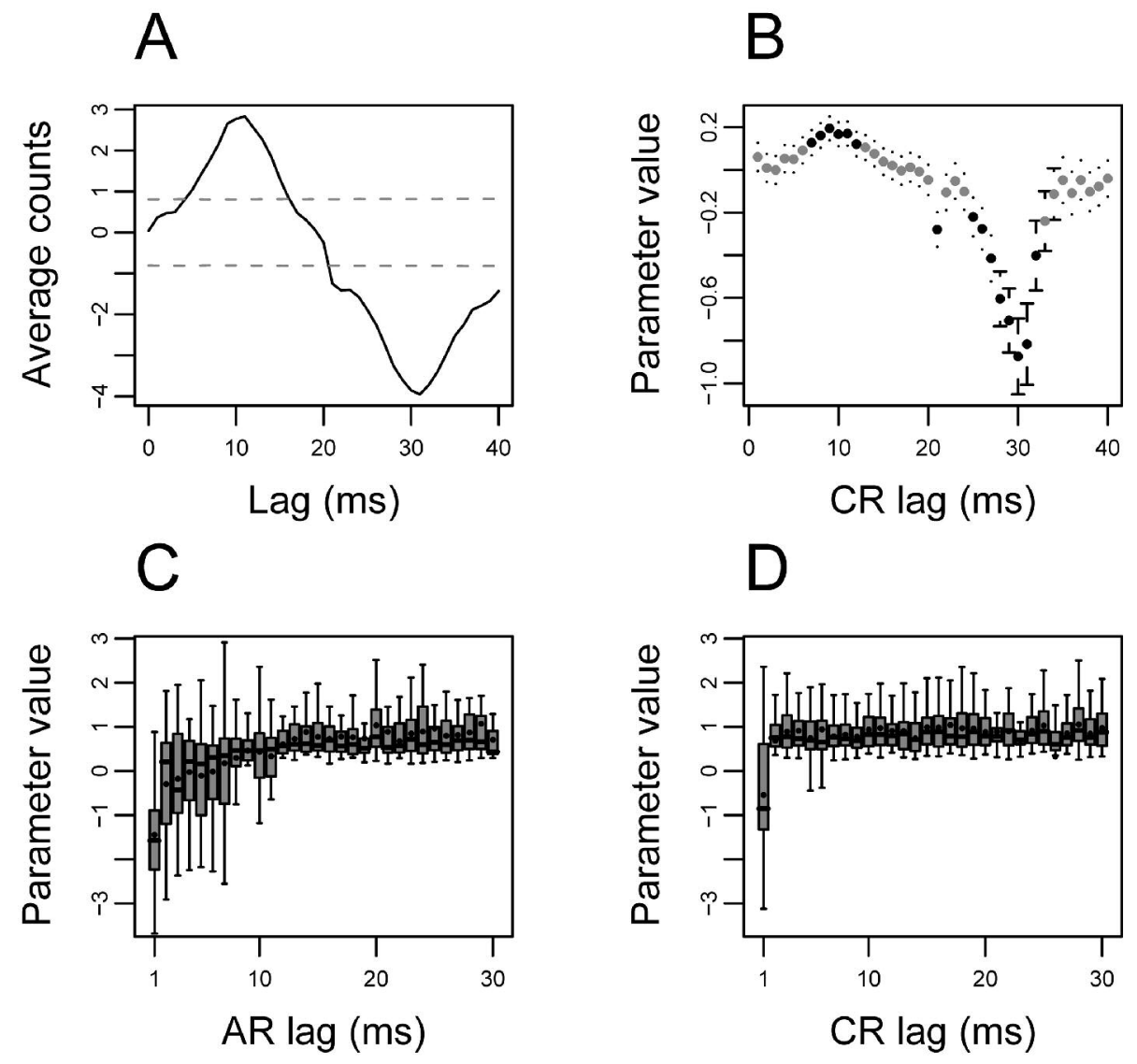

Figure 3. Model interpretation. A, Covariogram between a pair of simulated neurons. Dashed lines indicate significance. B, CR parameter values with standard errors (from Wald $\mathrm{Z}$ test) from the GLM fit for the same pair. The gray points are not significantly different from 0 . C, AR parameter values significantly different from 0 for hard and easy search models. The filled point is the mean, the horizontal bar is the median, the box delimits the interquartile range, and the whiskers extend to the point no more than 1.5 times the interquartile range. Outliers are not shown in the figure but are included in the analyses. $D$, The same for $C R$ parameters. $120 \times 109 \mathrm{~mm}(600 \times 600 \mathrm{DPI})$ 

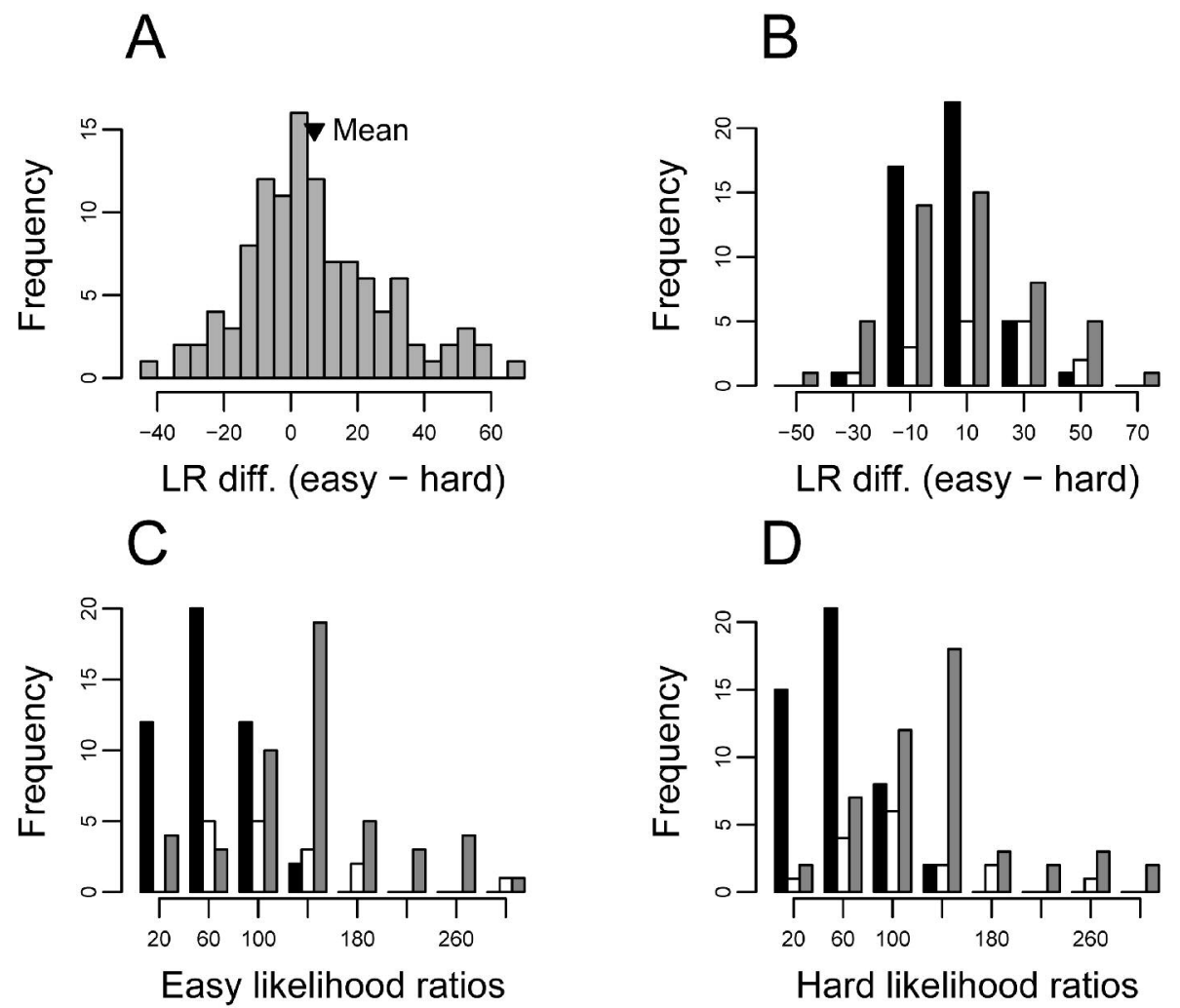

Figure 4. Likelihood ratio analysis. A, Histogram of likelihood ratio (LR) differences (easy minus hard) for each neuron). B, Histogram of LR differences split by neuron class (black: visual-related neurons; gray: visual-and-movement-related neurons; white: movementrelated neurons). C, Histogram of LRs in easy task models split by neuron class. $D$, Histogram of LRs in hard task models split by neuron class. 

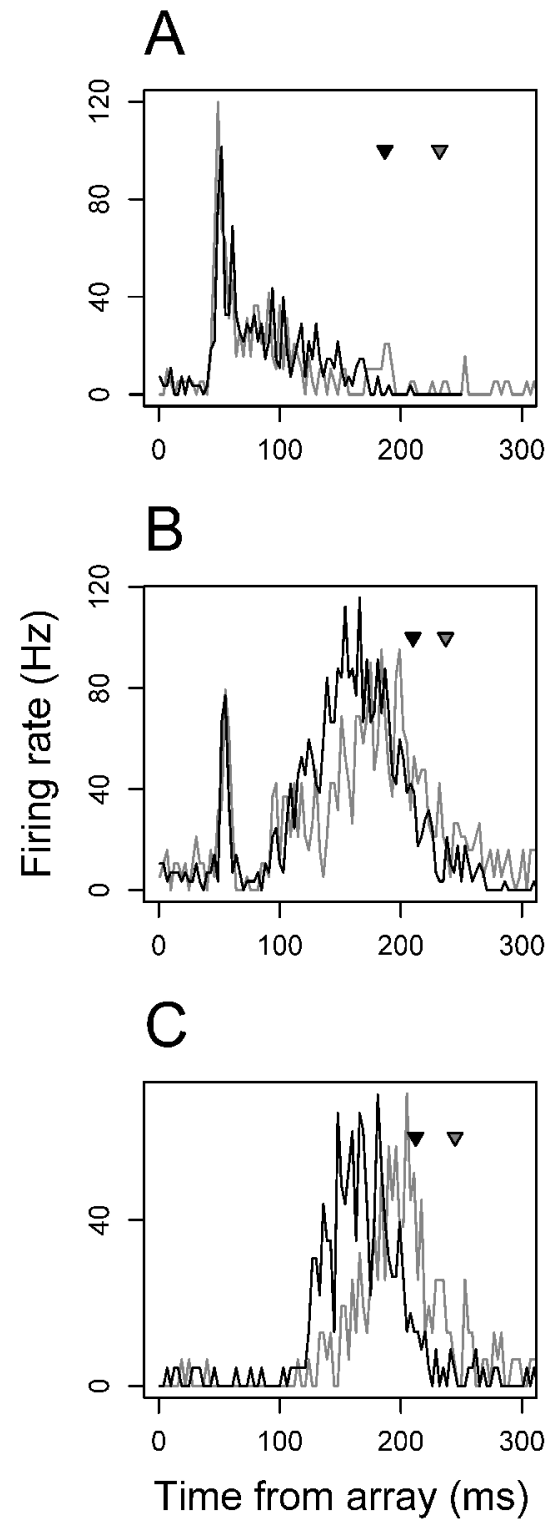

Figure 5. Example neurons of each class. A, PSTH for a visual-related neuron for the hard (gray) and easy (black) tasks aligned to array onset. B, The same for a visual-andmovement-related neuron. $C$, The same for a movement-related neuron. Bin size is $\mathbf{3} \mathbf{~ m s .}$ The black arrowhead denotes the mean saccade reaction time during the recording session for the easy task, the gray arrowhead for the hard task. $69 \times 177 \mathrm{~mm}(1200 \times 1200 \mathrm{DPI})$ 

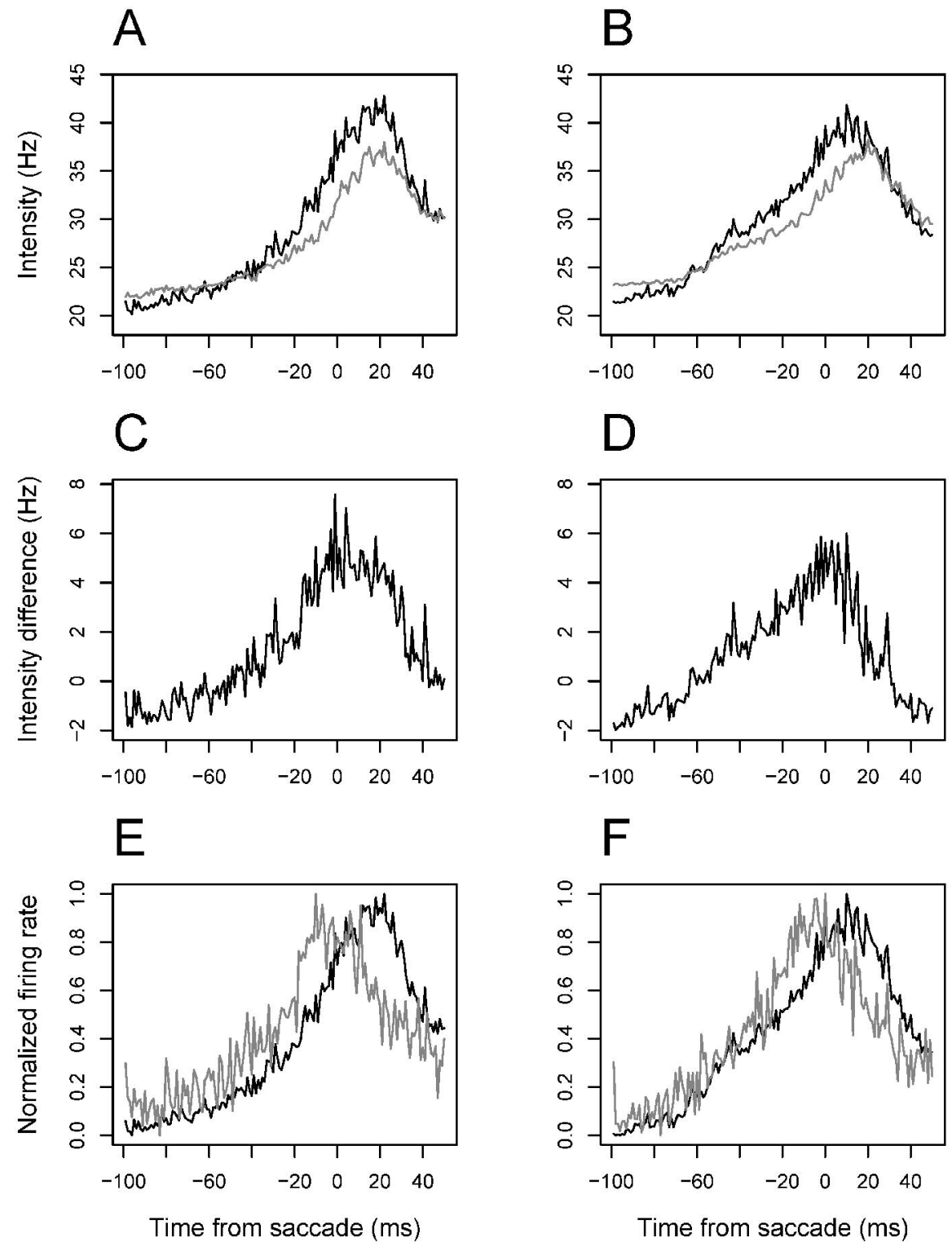

Figure 6. Mean instantaneous firing rate of movement-related neurons prior to saccade. A, The black curve is the mean intensity function for the $\mathbf{1 6}$ movement-related neurons during the hard search task, averaged over all trials for AR-CR models. The gray curve is the same for AR models. The curves are aligned to each trial's saccade. B, The same for AR-CR models (black) and AR models (gray) for the easy task. C, The difference between $A R-C R$ and $A R$ intensities for the hard task, i.e., the difference between the black and gray curves in A. D, The difference between AR-CR and AR intensities for the easy task. E, Comparison of intensities and PSTHs. Normalized mean intensity function (black curve) and PSTH (gray curve, $1 \mathrm{~ms}$ bins) for the hard task. F, The same for the easy task. $139 \times 177 \mathrm{~mm}(600 \times 600 \mathrm{DPI})$ 


\section{A}

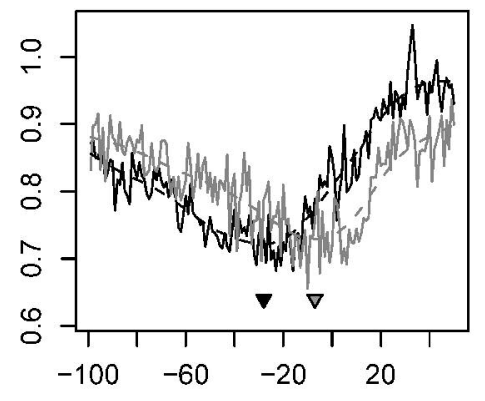

B
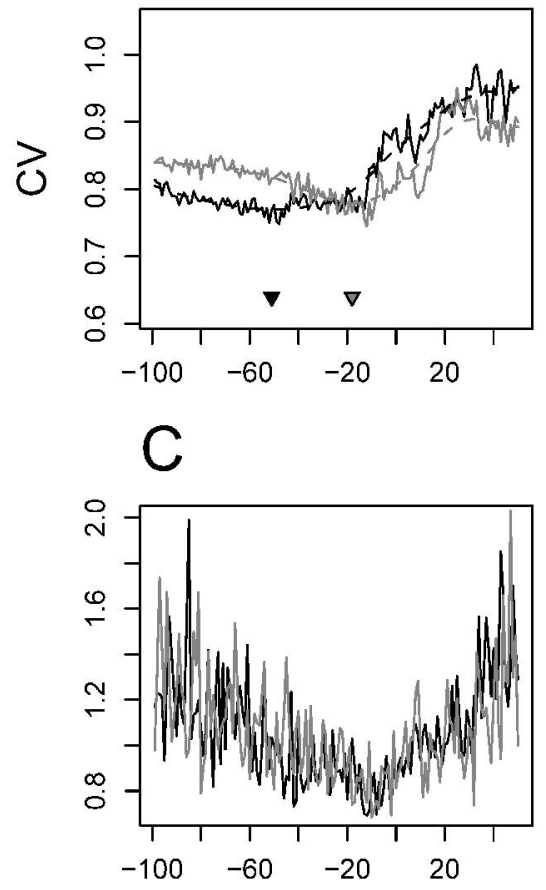

Time from saccade $(\mathrm{ms})$

Figure 7. Coefficient of variation of intensities and PSTHs. A, The gray curve is the coefficient of variation of the mean intensity functions for the movement-related neurons during the hard task, the black curve during the easy task, averaged over all trials for ARCR models. The dashed curves are smoothing splines, used to estimate the minima of the curves. Arrowheads denote these minima. B, The same for AR models. C, The gray curve is the coefficient of variation of the mean PSTH during the hard task, the black curve during the easy task.

$69 \times 177 \mathrm{~mm}(1200 \times 1200$ DPI $)$ 